\title{
Caracterización química como línea base para una gestión de cuenca, Bahía Drake
}

\author{
Chemical characterization as a baseline for watershed management, Drake Bay \\ John Diego Bolaños ${ }^{1}$ \\ Mayron Sancho Calvo ${ }^{2}$
}

Fecha de recepción: 25-01-2021

Fecha de aceptación: 30-09-2021

\begin{abstract}
Resumen
El artículo provee una línea base de conocimiento científico sobre iones cuantificados en cuerpos de agua dulce, en particular, de cinco ríos de la zona de Bahía Drake; esto para comprender los riesgos y vulnerabilidades del ecosistema, así como generar alertas en la protección del recurso hídrico. Se analizaron un total de 84 muestras representativas de los ríos Drake, Agujitas, Claro, Llorona y Sierpe, en tres campañas intensivas de muestreo, dos durante estación de verano y una en invierno, correspondientes a los años 2019 y 2020. Se determinaron los iones disueltos de cloruros, sulfatos, calcio, sodio, magnesio, potasio, zinc, plomo, cadmio, cobre, arsénico, manganeso y mercurio; para utilizarlos como indicadores de calidad ambiental y de salud humana. Los resultados destacan que la concentración de aniones en exceso proviene, principalmente, del impacto agropecuario en la zona de estudio, situación que es notoria en los ríos Drake, Llorona y Agujitas, en comparación con los ríos Claro y Sierpe; sin embargo, todos los ríos estudiados presentaron concentraciones iónicas de ríos sanos desde el punto vista químico, con una calidad aceptable una vez cotejados con la normativa nacional aplicable. La estación lluviosa mejora el equilibrio químico iónico de las sales disueltas en el agua, gracias al factor de dilución y a la capacidad de oxidación de la carga másica orgánica que transita sobre los ríos, lo cual favorece su oxigenación y acelera los procesos de depuración natural del río. Finalmente, no hay evidencia de metales pesados disueltos que favorezcan procesos de bioacumulación en los ecosistemas acuáticos estudiados.
\end{abstract}

Palabras clave: calidad del agua; caracterización química, agua superficial, administración de cuenca

\footnotetext{
1 Bachiller en Laboratorista Químico, Licenciado en Farmacia, Máster en Ciencias con énfasis en Gestión y Estudios Ambientales. Depto. Ciencias Naturales, Sede de Occidente, Universidad de Costa Rica, Costa Rica. Correo electrónico: john.bolanos@ucr.ac.cr

2 Estudiante de Licenciatura en Laboratorista Químico, Bachiller en Laboratorista Químico de la Sede de Occidente, Universidad de Costa Rica, Costa Rica. Correo electrónico: mayron.sancho@ucr.ac.cr
} 


\begin{abstract}
The article provides a baseline of scientific knowledge on quantified ions in freshwater bodies, in particular five rivers in the Drake Bay area; data to understand at a macro level the risks and vulnerabilities of the ecosystem, as well as to generate alerts in the protection of water resources. A total of 84 representative samples from the Drake, Agujitas, Claro, Llorona and Sierpe rivers were analyzed in three intensive sampling campaigns (two during the summer season and one in winter, corresponding to the years 2019 and 2020). The dissolved ions of chlorides, sulfates, calcium, sodium, magnesium, potassium, zinc, lead, cadmium, copper, arsenic, manganese and mercury were determined; to be used as indicators of environmental quality and human health. The results allow us to highlight that the excess anion concentration comes mainly from the impact of agricultural activity in the study area, a situation that is more evident in the Drake, Llorona and Agujitas rivers, compared to the Claro and Sierpe rivers; However, all the rivers studied present ionic concentrations that allow them to be classified from the chemical point of view, as healthy rivers with an acceptable quality, once they have been compared with the applicable national regulations. On the other hand, the rainy season improves the ionic chemical balance of the salts dissolved in the water, thanks to the dilution factor and the oxidation capacity of the organic mass load that transits over the rivers.

Finally, there is no evidence of dissolved heavy metals that favor bioaccumulation processes in the aquatic ecosystems under study.
\end{abstract}

Keywords: Water quality; chemical characterization, surface water, basin management

\section{Introducción}

En Costa Rica, una región rica en diversidad natural, el cuido de los ecosistemas es una responsabilidad compartida por todos los actores que componen el país; las instituciones públicas han fomentado el desarrollo de aportes científicos encausados hacia la prevención de los problemas de contaminación y la creación de estrategias que le permitan al territorio asegurar un desarrollo sostenible y sustentable. No es de extrañar que, recientemente, la Organización de las Naciones Unidas reconozca al país, su carácter de líder en la materia y lo premie con títulos como el de Campeones de la Tierra 2019 (ONU, 2019).

Como red arterial de una cuenca, los ríos y arroyos recogen mucha información vital de la zona por donde discurren; lo cual resume la calidad de sus aguas en sí mismos. Igualmente, nuevas perturbaciones y cambios con el uso del suelo tendrán una incidencia directa tanto a nivel biológico, como en las características físicoquímicas del agua (García-Avilés, 2002).

El presente artículo tiene como objetivo proveer a la población de Bahía Drake, y al país en general, una línea base de información química respecto a los afluentes que conforman la cuenca hidrográfica, para así permitir a investigadores, a la comunidad y al Estado, generar una adecuada respuesta a los problemas relacionados con la explotación de recursos naturales desmedidos.

El creciente aumento en la contaminación del recurso hídrico muchas veces no es percibido por la población, situación que, por "desconocimiento", puede traer consecuencias difíciles de revertir en el tiempo. Problemática ocasionada por el irrespeto a normativas ambientales justificadas desde la necesidad como medida de subsistencia, para alcanzar una mejora socioeconómica y de supuesto "bienestar" en una comunidad de difícil acceso, donde se brinda poco apoyo gubernamental y hay un lento desarrollo económico.

\section{Materiales y métodos}

La caracterización del agua en la zona de estudio es un aporte conjunto del Recinto de Grecia, el CIMAR, el CICANUM y las Escuelas de Microbiología, Ingeniería Civil, Topografía y Estadística; quienes apoyan a la comunidad de Bahía Drake con sus análisis a través de un proyecto de investigación denominado: Conservación de zonas de alta densidad de biodiversidad, por medio de la caracterización de contaminantes disueltos y sólidos suspendidos en el recurso hídrico e identificación de tecnologías en salud para las zonas costeras. Península de Osa. 
Sobre los puntos de muestreo: Son 84 muestras representativas de agua dulce, correspondientes a tres campañas intensivas de muestreo. Las primeras dos se realizaron en el año 2019, estación seca en el mes de febrero y lluviosa en el mes de Julio; la tercera se realizó en el año 2020, estación seca en el mes de febrero. Las muestras fueron tomadas a lo largo de cada río, específicamente en los ríos Drake, Agujitas, Claro, Llorona y Sierpe, en tres puntos específicos, con dos muestras por punto muestreado. El primer punto de muestreo se ubica en un sitio cercano al origen del río, identificado, en cada muestreo, con un "1", por ejemplo RD-1 que significa río Draque zona alta del río; el segundo ubicado en un punto medio del cauce, identificado con un "2", por ejemplo RLL2 que significa río Llorona, zona media a lo largo del cauce; y, finalmente, el último cercano a sus desembocaduras, identificado con un " 3 ", que correspondería a una muestra tomada en el Río Sierpe en la zona más baja, cercano a la costa, es decir RS-3, como lo muestra la Figura 1. Todos los puntos fueron referenciados con coordenadas para mantener sus ubicaciones en los muestreos.

Figura 1. Sitios de muestreo para la determinación química en muestras agua dulce en la zona del Pacifico sur de Costa Rica. Tomado y modificado de Google maps.

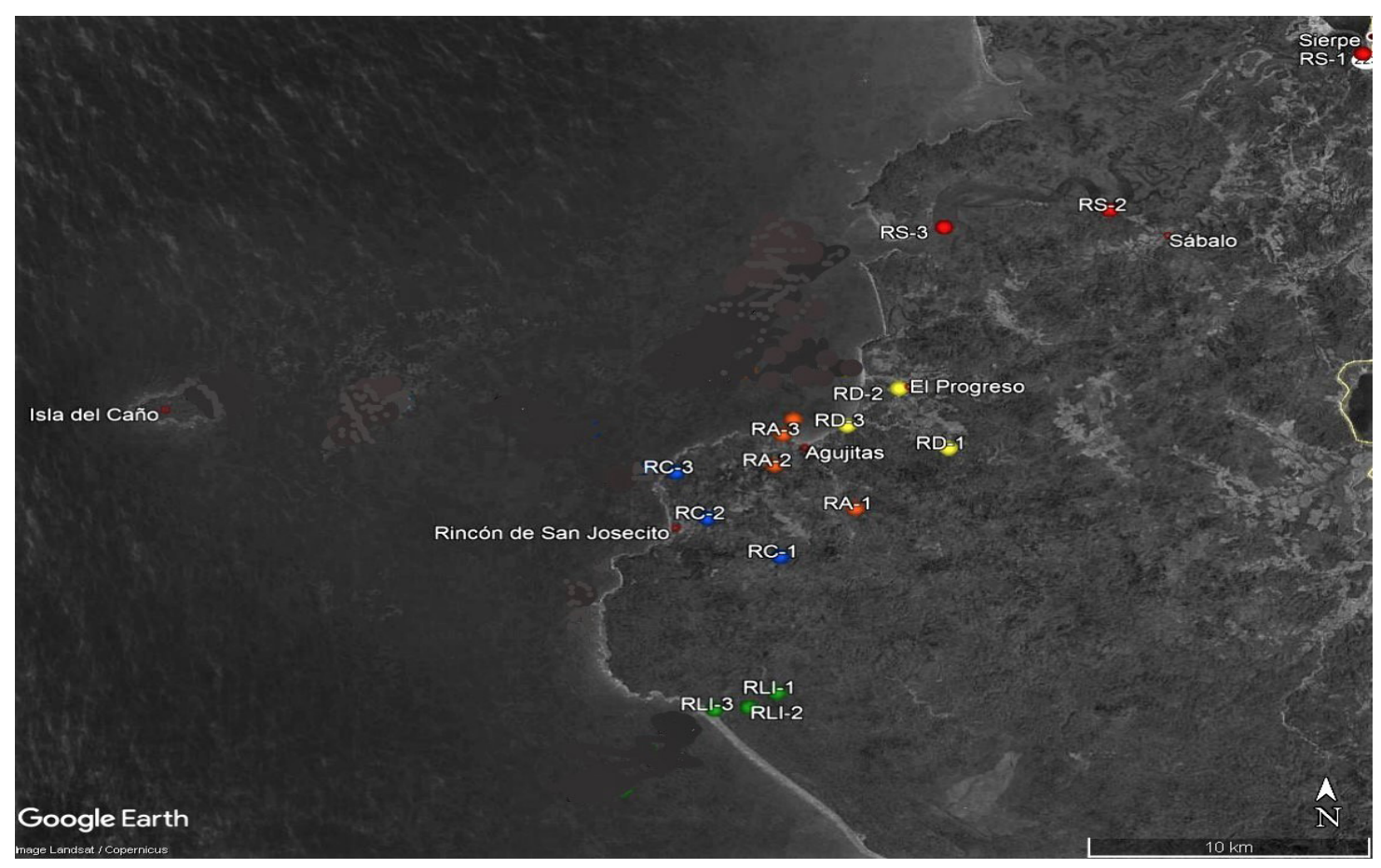

Procedimiento y determinación de muestras. Para realizar el proceso de toma de muestras se siguió la norma ISO 5667 Calidad del Agua (Internacional Standard, 2006). Dichas muestras fueron analizadas en el laboratorio de química del Recinto de Grecia, Universidad de Costa Rica, en línea con el procedimiento indicado por el Standard Metohods for the examination of water and wastewater, establecido por la Asociación Americana de salud pública por sus siglas en ingles APHA, 1992, 18 edición, pp 3-9, 3-12; se utilizó la técnica de cromatografía de iones y los procedimientos RUG-LQ-017 necesarios para cuantificar aniones, así como el procedimiento RUG-LQ-018 para la identificación y cuantificación de cationes, validados por el Laboratorio de Química del Recinto de Grecia mediante el proyecto No 540-B1-227 (2013). 
Para el análisis de los metales pesados $\mathrm{Cu}, \mathrm{Zn}, \mathrm{Pb}, \mathrm{Mn}$, Cr y Cd, se utilizó un espectrofotómetro de absorción atómica, modelo ICE 3000 marca ThermoScientific; además, se utilizó el acople al generador de hidruros VP10o marca ThermoScientific para el análisis de As y Hg. El procedimiento empleado fue el Standard Metohods for the examination of water and wastewater, establecido por la Asociación Americana de salud pública por sus siglas en ingles APHA, 1992, 18 edición, pp 3-9, 3-12 (AWWA, 1999).

La curva de calibración para cada metal estuvo compuesta por siete patrones, que abarcaron concentraciones de o,01 a 1,5 mg/L para el Zn, o,4 a $20 \mathrm{mg} / \mathrm{L}$ para el $\mathrm{Pb}$, o,025 a $3 \mathrm{mg} / \mathrm{L}$ para el Cd, o,05 a 1,5 mg/L para Cu, o,001 a 0,06 mg/L para As, o,2 a $8 \mathrm{mg} / \mathrm{L}$ para Mn y o,1 a $5 \mathrm{mg} / \mathrm{L}$ para $\mathrm{Hg}$; una curva de aniones que se realizó, contiene seis patrones en un coctel con $\mathrm{Cl}^{-}$y SO$~_{4}^{-2}$, que abarcaron concentraciones de 0,4 a $14 \mathrm{mg} / \mathrm{L}$; otra curva de calibración de siete patrones utilizando un coctel con los cationes $\mathrm{Ca}^{2+}, \mathrm{Mg}^{2+}, \mathrm{Na}^{+}$y K $\mathrm{K}^{+}$, que abarcaron concentraciones de 0,4 a $45 \mathrm{mg} / \mathrm{L}$. Esto fue determinado en un equipo de cromatografía de iones modelo Dionex, marca ThermoScientific, con las columnas modelo CS12A Thermo para el análisis de cationes y AS23 Thermo para el análisis de aniones.

Análisis de la información. Se calcularon promedios de concentraciones para los iones de estudio. La gráfica unidimensional fue necesaria como herramienta para analizar el comportamiento de los parámetros y visualizar características destacables. La estadística inferencial se utilizó para estudiar fenómenos aleatorios desconocidos. Finalmente, se utilizaron normas, reglamentos e investigaciones, para corroborar y comparar los datos obtenidos, respecto a los parámetros para la calidad del agua.

\section{Discusión y resultados}

El estudio de estos ríos, aporta información valiosa para conocer la calidad hídrica que tiene el ecosistema y sus pobladores; estos indicadores de la calidad química del agua funcionan, posteriormente, para establecer, desde el punto de vista científico, la vulnerabilidad que tiene el recurso por efectos antropogénicos o naturales, así como para que entidades públicas y privadas (ASADA, Municipalidad, Asociación de Desarrollo, entre otras) tomen decisiones utilizando la información científica.

En las últimas dos décadas, la zona de estudio ha presentado un aumento demográfico y turístico importante que, sin duda, impacta en la calidad del agua superficial. Cuando se habla de la calidad del agua, se entiende que las características químicas, físicas y biológicas del agua en todos sus estados, son aptas para mantener los ecosistemas naturales o para un uso particularmente antropogénico. Dicha calidad se puede ver disminuida o alterada por diferentes contaminantes; la evaluación de la calidad del agua es, por tanto, indispensable para determinar cuál es el uso que se le puede dar al agua (Mainstone y Parr, 2002)

Es importante recordar que las características del agua varían, de ahí que para evaluar su calidad se deban considerar las condiciones del entorno, así como los factores que pueden alterarla, con miras a tomar las medidas necesarias para mejorar su estado (Ashraf et al., 2010). La normativa nacional contempla una serie de especies iónicas de importancia para el control de la calidad del agua, en ella se detallan las sales iónicos que pueden jugar un papel importante en el metabolismo de las plantas y animales, es decir que influyen en el medioambiente y por ende su calidad y en el uso de la misma (Cravo, 2017).

\subsection{Cationes mayoritarios: Calcio, Magnesio, Sodio y Potasio}

Su cuantificación en aguas naturales es importante como indicadores de salud humana y ambiental; por ejemplo, la concentración de iones sodio y potasio se asocian con el transporte e intercambio iónico en los cuerpos de agua, un proceso esencial para el desarrollo de los ecosistemas biológicos, igual sucede si se piensa en su consumo o uso a nivel fisiológico por parte del ser humano. El calcio y magnesio puede ser estudiados según el interés particular, se relaciona con la dureza del agua, por ende una alta concentración en el agua sin duda afectará tuberías, utensilios metálicos, termoduchas, así como maquinaria industrial; por otra parte, el calcio actúa sobre 
el crecimiento y dinámica de las poblaciones de fauna y flora de cuerpos de agua, y el magnesio es necesario y debe estar presente en las plantas que tienen clorofila (Palter y Ballestero, 2007). Si se piensa en salud humana, el consumo prolongado de aguas duras conduce a serios problemas de salud, entre los que se pueden citar procesos de insuficiencia renal, caries dentales y deficiencias óseas como la osteopenia (Martinez de Victoria, 2016).

\section{Caracterización del ion Calcio $\left[\mathrm{Ca}^{2+}\right]$ en agua} superficial. La Figura 2 denota que la mayoría de los puntos muestreados se encuentran por debajo de los 100 $\mathrm{mg} / \mathrm{L}$, límite máximo admisible para la concentración del ion $\left[\mathrm{Ca}^{2+}\right]$ por el Reglamento para la calidad del agua potable, $\mathrm{N}^{\circ} 38924$-S en muestras de agua superficial, a excepción de los puntos RLL-3, RLL-2, RA-3 y RD-3 de la primera campaña y RD-3, RA-3 de la tercera campaña de muestreo (ambas realizadas en estación seca). Estos corresponden, en su mayoría, a zonas cercanas a las desembocaduras y que, por ende, disparan la concentración del catión debido a la intrusión salina provocada por las mareas, ya que el agua de mar generalmente contiene concentraciones mucho más elevadas de calcio que el agua dulce superficial (Arellano, F. y Vargas, 2000).

Las concentraciones bajas del ion calcio en las zonas altas y medias de las cuencas permite catalogarla como aguas blandas, atribuible a suelos ígneos; la presencia de este ion en el agua varía de acuerdo a la proximidad con la fuente natural, típicamente en aguas superficiales se puede encontrar en concentraciones de 20 a $100 \mathrm{mg} / \mathrm{L}$ (Beita, 2008). Se destaca, además, que la precipitación presente durante la temporada de invierno provoca una dilución en las concentraciones del ion (Dávila \& Valdés, 2015).

Figura 2. Caracterización del ion $\left[\mathrm{Ca}^{2+}\right]$ en muestras de agua superficial en los ríos Sierpe, Drake, Agujitas, Claro y Llorona, en tres muestreos realizados durante 2019-2020. La línea horizontal indica la concentración máxima admisible por el Reglamento para la calidad del agua potable No 38924-S.

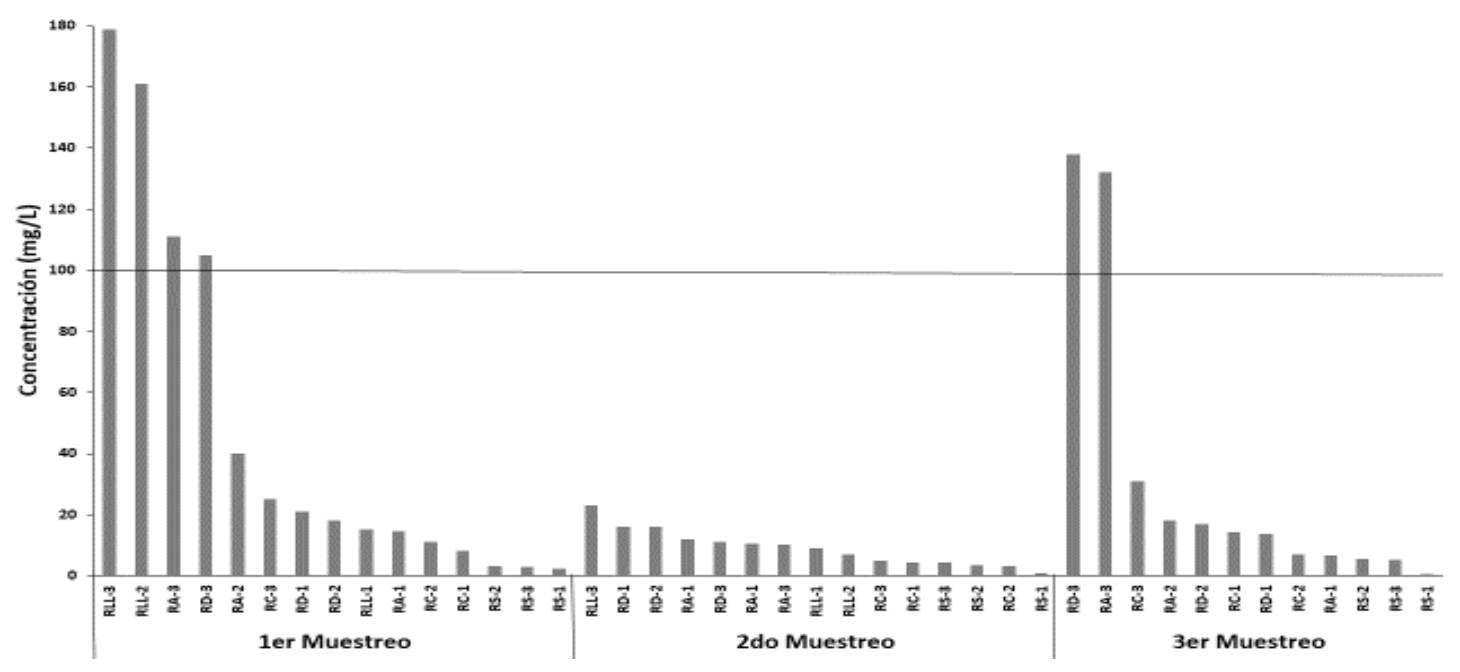

Caracterización del ion Magnesio $\left[\mathrm{Mg}^{2+}\right]$ en agua superficial. La mayoría las muestras presentan menos de $50 \mathrm{mg} / \mathrm{L}$ de magnesio, límite máximo admisible para la concentración del ion $\left[\mathrm{Mg}^{2+}\right]$ por el Reglamento $\mathrm{N}^{\circ} 38924-\mathrm{S}$, lo que permite catalogarla como agua de calidad respecto la concentración del ion, con excepción de los puntos RLL3, RLL-2, RA-3, RD-3 de la primera y tercera campaña de muestreo. De acuerdo con el reglamento para la evaluación y clasificación de Cuerpos de agua superficiales de Costa Rica (MINAE, 2007), el valor de 8,7 mg/L sería un valor recomendado para la conservación y desarrollo de la vida acuática. Los puntos RLL-3, RD-3, RA-3 y RC-3 corresponden a zonas cercanas a desembocaduras de los ríos al mar; además, fueron sitios muestreados en estación seca; no obstante, existe normativa ambiental de calidad de aguas superficiales y zonas costeras que señalan, como valor máximo aceptable en cuerpos hídricos superficiales y aguas costeras, una concentración del ion $\left[\mathrm{Mg}^{2+}\right]$ de 
$300 \mathrm{mg} / \mathrm{L}$ (Ministerio de ambiente y recursos naturales, 2012). Se atribuye, entonces, dicho comportamiento de cambio abrupto a la influencia de la marea en las costas en las desembocaduras de los ríos Llorona, Drake, Agujitas y Claro, lo cual induce un aumento significativo en las concentraciones del ion, debido a que según Arellano, F. y Vargas (2000), el agua de mar contiene concentraciones más altas de magnesio en comparación con las aguas continentales.

\section{Caracterización del ion Sodio [ $\left.\mathrm{Na}^{+}\right]$en muestras} de aguas superficiales. En la figura 3 se muestra que la mayoría de los sitios analizados se encuentran por debajo de los $200 \mathrm{mg} / \mathrm{L}$, límite máximo admisible para la concentración del ion, según el Reglamento $\mathrm{N}^{\circ} 38924-\mathrm{S}$, a excepción de los sitios RLL-3, RLL-2, RA-3, RD-3 de la primera campaña y los sitios RA-3, RC-3, RS-3, RD-3 tercera campaña de muestreo. Concentraciones altas de sodio influyen de forma importante en el agua para riego y hasta para consumo humano ya que, inclusive, pueden dañar las funciones renales, cardiacas y circulatorias. Sin embargo, no hay referencia regulatoria para el impacto que pueda causar en la conservación y preservación de la vida acuática de ecosistemas de agua dulce; no obstante, se sabe que las concentraciones del ion pueden llegar a ser mayores a los $10000 \mathrm{mg} / \mathrm{L}$ en agua de mar (Beita, 2008).
El ámbito de concentraciones fue de 1,8 mg/L a 9846 $\mathrm{mg} / \mathrm{L}$, en donde el punto de muestreo localizado en la desembocadura del río Llorona (RLL-3) mostró la mayor concentración promedio $9789 \mathrm{mg} / \mathrm{L}$ que se atribuye a la influencia de la marea que, por difusión, aumenta la concentración del agua salada en el agua dulce de las desembocaduras. Por otro lado, se destaca la influencia de la estación lluviosa (segunda muestreo), en donde, con excepción del río Llorona, las concentraciones del ion son visiblemente más bajas, en un rango de 3,1 mg/L a 190 $\mathrm{mg} / \mathrm{L}$ en comparación con el primer y tercer muestreo, debido al factor de dilución que sufre el ion con la lluvia.

La concentración de sodio presentó en promedio $31,6 \mathrm{mg} / \mathrm{L}$ en las partes altas y medias de las cuencas analizadas, una concentración mayor con respecto a un estudio realizado por Barahona y Beita en el 2004 al río Rincón, cerca de la zona de estudio, lo cual determinó una concentración promedio de $7,3 \mathrm{mg} / \mathrm{L}$ para dicha cuenca (Barahona y Beita, 2011). Tal situación denota su vulnerabilidad a procesos de pérdida de suelo, provocada por procesos erosivos debido a la ganadería extensiva, una de las principales actividades agropecuarias en la zona cercana al golfo.

Figura 3. Caracterización del ion $\left[\mathrm{Na}^{+}\right]$en muestras de agua superficial en los ríos Sierpe, Drake, Agujitas, Claro y Llorona, en tres muestreos realizados entre 2019-2020. La línea horizontal indica la concentración máxima admisible por el Reglamento para la calidad del agua potable No 38924-S.

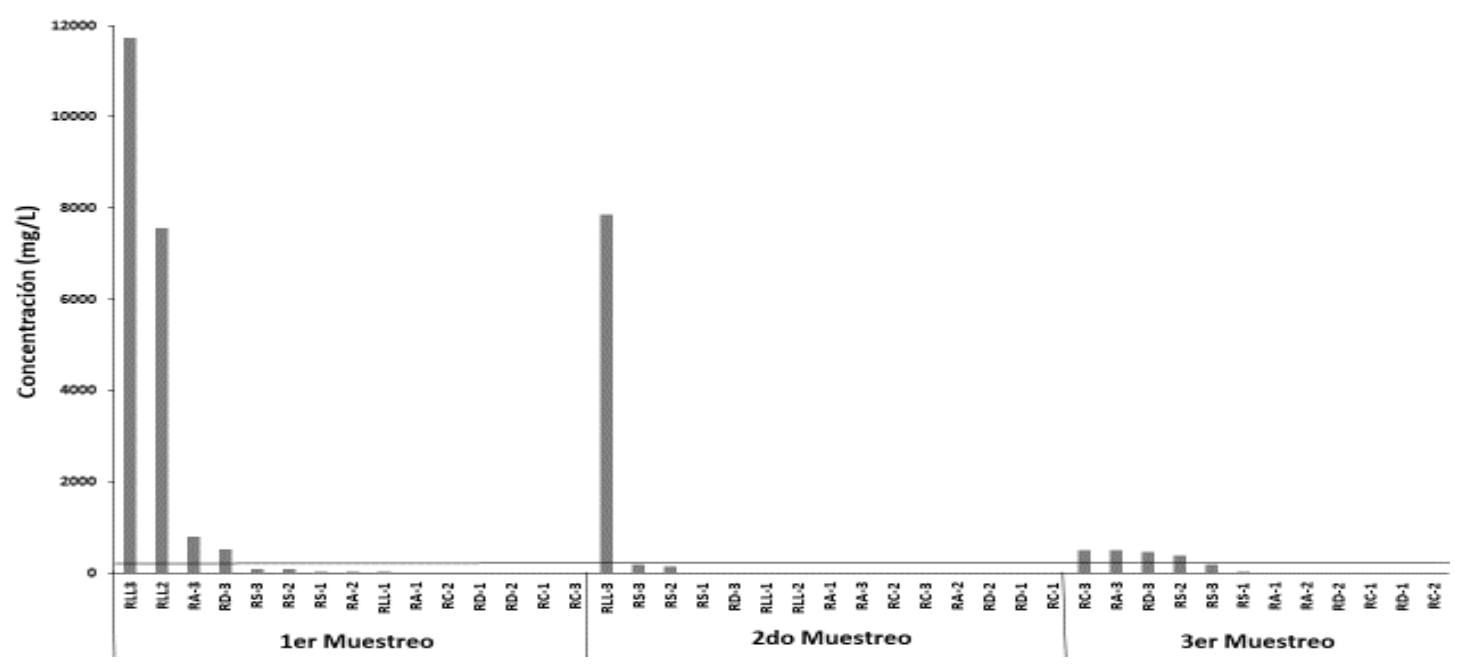




\section{Caracterización del ion Potasio [ $\left.\mathrm{K}^{+}\right]$en agua superficial.} La concentración de Potasio en la mayoría de los puntos de muestreo se encuentran por debajo de los $10 \mathrm{mg} / \mathrm{L}$, límite máximo admisible para la concentración del ion $\left[\mathrm{K}^{+}\right]$por el Reglamento $\mathrm{N}^{\circ} 38924-\mathrm{S}$ en muestras de agua superficial, a excepción de los puntos correspondientes a las desembocaduras de los ríos de estudio.

El ámbito de concentraciones obtenido fue de o,9 $\mathrm{mg} / \mathrm{L}$ a $447 \mathrm{mg} / \mathrm{L}$. El punto de muestreo localizado en la desembocadura del río Llorona (RLl-3) mostró, igualmente, la mayor concentración promedio 447 $\mathrm{mg} / \mathrm{L}$; consistentemente, el estudio refleja que la estación de invierno influye visiblemente en la reducción de la concentración del ion por dilución, debido a que las aguas de lluvia son captadas por los cuerpos de agua, lo cual produce un aumento en el aforo del río y, por lo tanto, una dilución de las especies químicas presentes en el cuerpo de agua (Dávila y Valdés, 2015). Las concentraciones más elevadas del ion obtenidas, se dieron en zonas de costas, donde la intrusión salina provocada por la difusión (LazoPáez y Solís-Castro, 2019), genera el cambio abrupto. Si se excluyen las desembocaduras en estación de verano, en general, la concentración del ion $\left[\mathrm{K}^{+}\right]$presentó un promedio de 4,8 mg/L en las partes altas y medias de las cuencas analizadas, una concentración mayor con respecto a un estudio realizado en el 2004 al río Rincón, que se ubica cerca de la zona de estudio y el cual arrojó un valor promedio por debajo de los o,89 mg/L (Beita, 2008); ambos estudios presentan una concentración inferior al máximo recomendado según normativa citada.

\subsection{Caracterización del ion Sulfato $\left[\mathrm{SO}_{4}{ }^{2-}\right]$ en agua superficial}

Este ión es una de las sustancias químicas que favorece la eutroficación en los ríos, lagos y costas marinas, teniendo como fuente de producción diversos procesos antrópicos; la erosión, escorrentía y la lixiviación son las principales vías para el transporte del anión, desde el ecosistema terrestre al acuático, y su existencia en aguas superficiales está relacionada con las emisiones de dióxido de azufre provocado por emisiones de fuentes móviles e industriales; no obstante, los sulfatos son abundantes en la naturaleza, su concentración en el agua varía por centenas de miligramos por litro; en el país depende, principalmente, de la presencia de sulfato de hierro existente en el suelo y lechos rocosos de ríos y océanos (Mainstone y Parr, 2002).

De acuerdo con el Reglamento para la Calidad del Agua Potable $\mathrm{N}^{\circ} 38924-\mathrm{S}$, se estima como valor alerta para sulfatos una concentración que no supere los $250 \mathrm{mg} / \mathrm{L}$. Es importante recordar que su forma iónica afecta al ser humano debido a su efecto laxante, cuando se supera una ingesta de $100 \mathrm{mg} / \mathrm{L}$ de sales como sulfato de sodio o sulfato de magnesio disueltas; lo que conlleva también a efectos secundarios como la deshidratación (BolañosAlfaro et al., 2017).

Las determinaciones del ion $\left[\mathrm{SO}_{4}{ }^{2-}\right]$ muestran que la mayoría de los puntos donde se cuantificó se encuentran por debajo del límite máximo admisible, a excepción de los puntos RLL-3 RLL-2 del primer muestreo y los puntos RA3, RD-3 correspondientes al tercer muestreo, ver Figura 4. Sin embargo, en contraste con la normativa ambiental de calidad de aguas superficiales y zonas costeras, en los ríos en preservación, con actividades portuarias y navegación en zonas costeras, la concentración del sulfatos puede llegar a superar los $5000 \mathrm{mg} / \mathrm{L}$ (Ministerio de ambiente y recursos naturales, 2012); aún así, para su utilización en riego, agricultura u otros usos, prevalece el reglamento para la evaluación y clasificación de cuerpos de agua superficiales de Costa Rica (MINAE, 2007), que, como se mencionó, establece un valor máximo de $250 \mathrm{mg} / \mathrm{L}$.

El aumento en la concentración del anión en desembocaduras de los ríos se atribuye, al igual que en los iones anteriores, principalmente, a la influencia de la marea; se sabe que el agua de mar contiene minerales muy ricos en sales de azufre, aun cuando existen otras fuentes de contaminación como lo son la combustión de materia orgánica, la descomposición o la emisión de volcanes (Grunberger y Janeau, 1991). La estación lluviosa, por otra parte, facilita la dilución de sus sales disueltas; no obstante, existen factores como la ganadería y la agricultura que pueden incrementar la concentración del anión en agua. En la zona se observaran grandes extensiones de suelo cultivadas con palma, así como el uso indiscriminado de fertilizantes a base de sulfato de 
amonio o sulfato de potasio que, gracias a los procesos de escorrentía, generan un constante lavado del minerales del suelo, lo que posibilita una contaminación con sulfatos (Casado y Calvo et al., 2013).

Excluyendo las desembocaduras, la concentración del ion $\left[\mathrm{SO}_{4}^{2-}\right]$ presentó, en promedio, una concentración de 5,4 $\mathrm{mg} / \mathrm{L}$ en la parte alta y media de los ríos de estudio, una concentración menor con respecto al estudio realizado por Beita en el 2004 en la parte alta del río Rincón cerca de la zona de estudio, que determinó una concentración promedio de los 7,6 mg/L para dicha cuenca (Beita, 2008), con lo cual se puede suponer que la calidad de los ríos respecto al ión es buena.

Figura 4. Caracterización del ion $\left[\mathrm{SO}_{4}{ }^{2-}\right]$ en muestras de agua superficial en los ríos Sierpe, Drake, Agujitas, Claro y Llorona, en tres muestreos realizados entre 2019-2020. La línea horizontal indica la concentración máxima admisible por el Reglamento para la calidad del agua potable No 38924-S.

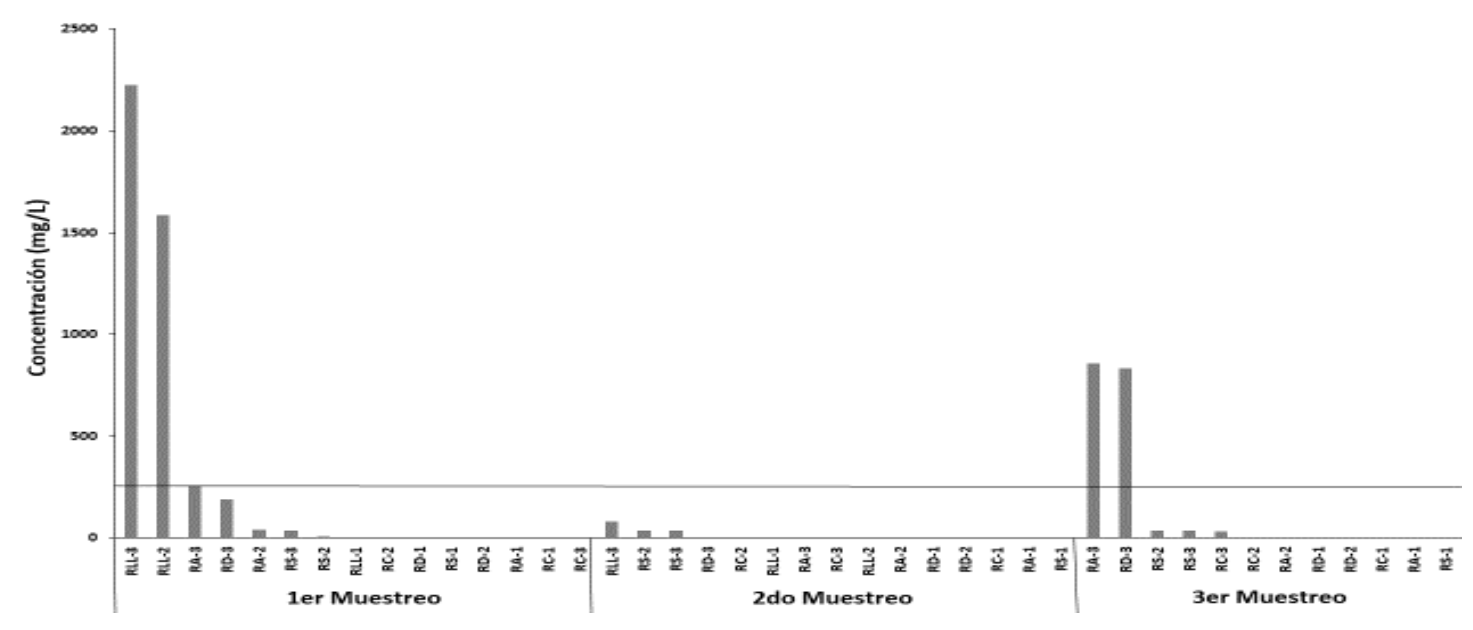

\subsection{Caracterización del ion Cloruro $\left[\mathrm{Cl}^{-}\right]$en agua superficial}

El cloruro es una especie iónica importante en el estudio de las aguas superficiales; presenta concentraciones variables dependiendo de las características de los terrenos por donde siga el curso del río, y según su cercanía a desembocaduras al mar o la posibilidad de intrusión salina en lechos subterráneos con alta explotación de agua, los cuales afectan los niveles freáticos (Alvarado et al., 2015). En zonas altas de los ríos de estudio, existe una cobertura forestal importante, sin embargo, a medida que los ríos se acercan a la desembocadura es notorio el desarrollo de actividades agropecuarias como la ganadería extensiva y la agricultura, y, por consiguiente, disminuye dicha cobertura.
Un aumento en la concentración de cloruros, en aguas subterráneas se achaca a factores de intrusión salina y procesos de lavado del suelo por escorrentía, así como a la contaminación con aguas residuales; el contenido de cloruros no debe superar los $60 \mathrm{mg} / \mathrm{L}$ en aguas para consumo humano, debido a que cambia, entre otros aspectos, las propiedades organolépticas del agua, entre ellas el sabor (Costa Rica Poder Ejecutivo, 2015). También, su existencia en espejos de agua propicia una reacción fotoquímica natural que induce la producción de bromatos, sustancia altamente tóxica y cancerígena para el ser humano y otras especies. Su elevada concentración daña estructuras metálicas provocando corrosión; en definitiva, perjudican el crecimiento vegetal en aguas superficiales, provocando procesos de clorosis y hasta necrosis en las estructuras celulares de los ecosistemas naturales de agua dulce (Stewart \& Elliott, 1996). 
La Figura 5 denota una la concentración de cloruro por debajo de los $250 \mathrm{mg} / \mathrm{L}$, límite máximo admisible según el Reglamento $\mathrm{N}^{\circ} 38924-\mathrm{S}$, a excepción de los puntos cercanos a la costa, en donde se supera significativamente lo normado, incluso, excede la normativa ambiental de calidad de aguas superficiales y zonas costeras para ríos, en preservación con actividades portuarias y abastecimiento industrial en zonas costeras (Ministerio de ambiente y recursos naturales, 2012).

Es importante tomar en cuenta que el anión cloruro es uno de los iones mayoritarios más difundidos en aguas naturales, su contaminación puede obedecer al ingreso de aguas residuales de origen antropológico al cause (Rosas, 2001). Su aumento, conforme se acerca a la costa marina, se justifica, principalmente, por la influencia de la marea, que promueve un aumento significativo debido a que el agua de mar contiene concentraciones muy altas de cloruros (Grunberger y Janeau, 1991).
Las concentraciones de todos los puntos de muestreo tomados en la segunda campaña (exceptuando el río Llorona y Sierpe) son concentraciones muy bajas, con un rango de $0,9 \mathrm{mg} / \mathrm{L}$ a $27 \mathrm{mg} / \mathrm{L}$, lo que permite catalogar el agua de los ríos como agua de buena calidad, sin embargo, el primer y tercer muestreo arrojó concentraciones altas del anión, lo cual cambió dicho criterio de calidad, de ahí que se excluyan los resultados de las desembocaduras en los cinco ríos para tal consideración. La concentración del anión presenta un promedio de $36 \mathrm{mg} / \mathrm{L}$ en las partes altas y medias de los ríos, una concentración superior con respecto al estudio realizado por Beita en el 2004 en la parte alta del río Rincón cerca de la zona de estudio, determinando una concentración promedio de o,6 mg/L para dicha cuenca (Beita, 2008); pero siempre inferior a lo normado, lo cual permite considerar los ríos de estudio con buena calidad respecto al indicador químico en dichas áreas.

Figura 5. Caracterización del ion [Cl-] en muestras de agua superficial en los ríos Sierpe, Drake, Agujitas, Claro y Llorona, en tres muestreos realizados entre 2019-2020. La línea horizontal indica la concentración máxima admisible por el Reglamento para la calidad del agua potable No 38924-S.

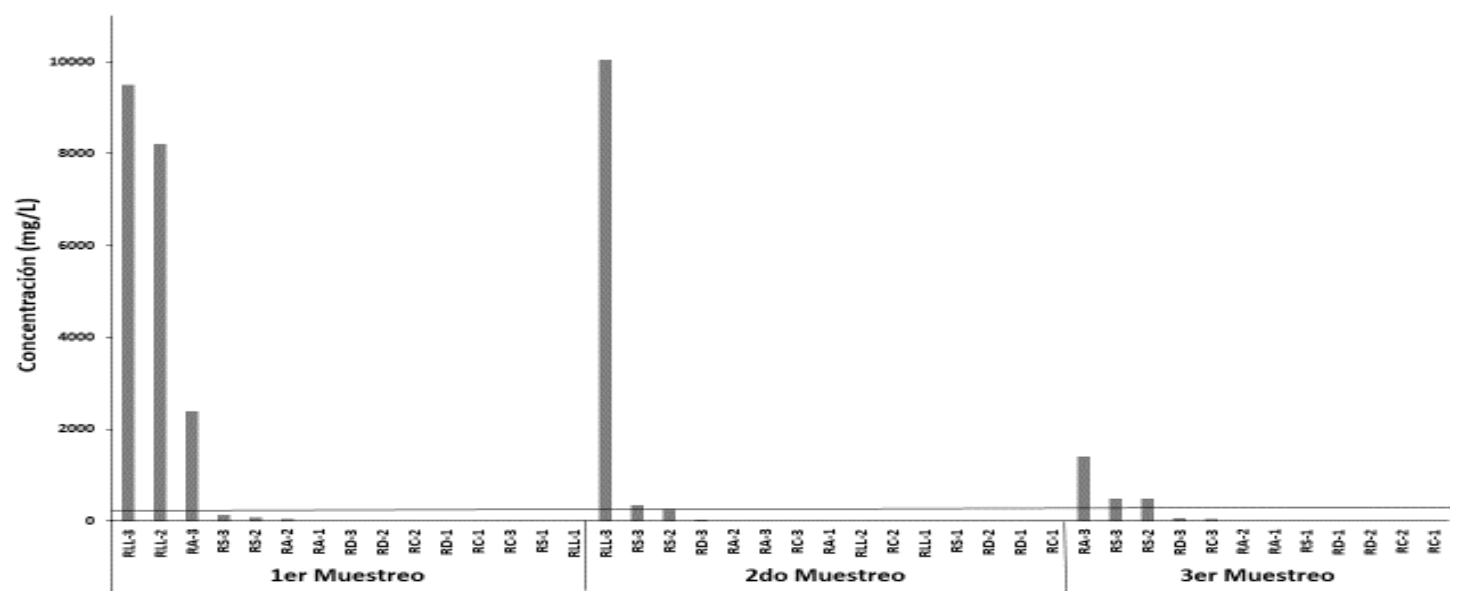

\subsection{Caracterización de metales pesados y otros elementos minoritarios}

La calidad del agua se afecta si existe contaminación con metales pesados u excesos de iones minoritarios como zinc, hierro, manganeso, cobre, mercurio, plomo, cromo, arsénico, cadmio, entre otros. En aguas superficiales y subterráneas, también llamadas aguas continentales, su existencia se convierte en un problema de importancia para la salubridad y la conservación ambiental. Tal situación ocurre por uso incorrecto y excesivo de agroquímicos, como los fertilizantes, que disparan dramáticamente las concentraciones de metales pesados en agua (Wongsasuluk et al., 2014). Con el tiempo, los metales pesados se biocumulan y alcanzan niveles tóxicos en los suelos agrícolas, lo cual contamina el primer eslabón 
de la cadena trófica; su capacidad potencial de acumularse en el suelo y superficies, así como la posibilidad de ser arrastrados por escorrentía, filtración y erosión, les permite alcanzar cuerpos de agua continentales, hecho que provoca adversidad para la cadena alimenticia (Wongsasuluk et al., 2014).

Analizar la concentración de los metales pesados y micronutrientes presentes en el ecosistema acuático del pacifico sur permite obtener una fotografía actual y observar perturbaciones naturales o antrópicas que pudiesen existir, así como conocer el efecto directo sobre el ecosistema (Morales-Ramírez et al., 2015)

Determinación de zinc (Zn). Mostró una concentración muy por debajo de los 3,o mg/L, límite máximo admisible para la concentración del Zn por el Reglamento $\mathrm{N}^{\circ} 38924-\mathrm{S}$. El ámbito de concentraciones obtenido fue de $0,02 \mathrm{mg} / \mathrm{L}$ a 0,07 mg/L. La concentración promedio fue de $0,04 \mathrm{mg} / \mathrm{L}$; valor que se encuentra dentro de lo permitido por la normativa ambiental de calidad de aguas superficiales y zonas costeras para ríos en preservación, con actividades portuarias, y abastecimiento industrial en zonas costeras, donde se establece como límite máximo de concentración de Zn en 0,05 mg/L (Ministerio de ambiente y recursos naturales, 2012). Por otro lado, de acuerdo con el reglamento para la evaluación y clasificación de cuerpos de agua superficiales de Costa Rica (MINAE, 2007), se establece un valor de $0,5 \mathrm{mg} / \mathrm{L}$ como valor máximo recomendado para la conservación y desarrollo de la vida acuática; todas las muestras analizadas se encuentran con concentraciones inferiores a este valor.

En un estudio realizado por Herrera, Rodríguez, Rojas, Herrera y Chaves durante los años 2006 y 2010, después de un análisis químico de la calidad de las aguas superficiales de la subcuenta del río Virilla (Costa Rica), se determinó una concentración de hasta $0,37 \mathrm{mg} / \mathrm{L}$ en la zona de la microcuenca de Torres (Valverde, 2013); ello obedece al fuerte impacto sobre los ecosistemas y la contaminación del agua superficial que sufre esta zona, fenómeno característico en muchas microcuencas del valle central.

\section{Figura 6. Concentración de Zinc en muestras de agua superficial en los ríos Sierpe, Drake, Agujitas, Claro y Llorona, en tres campañas de muestreo realizados entre 2019-2020.}

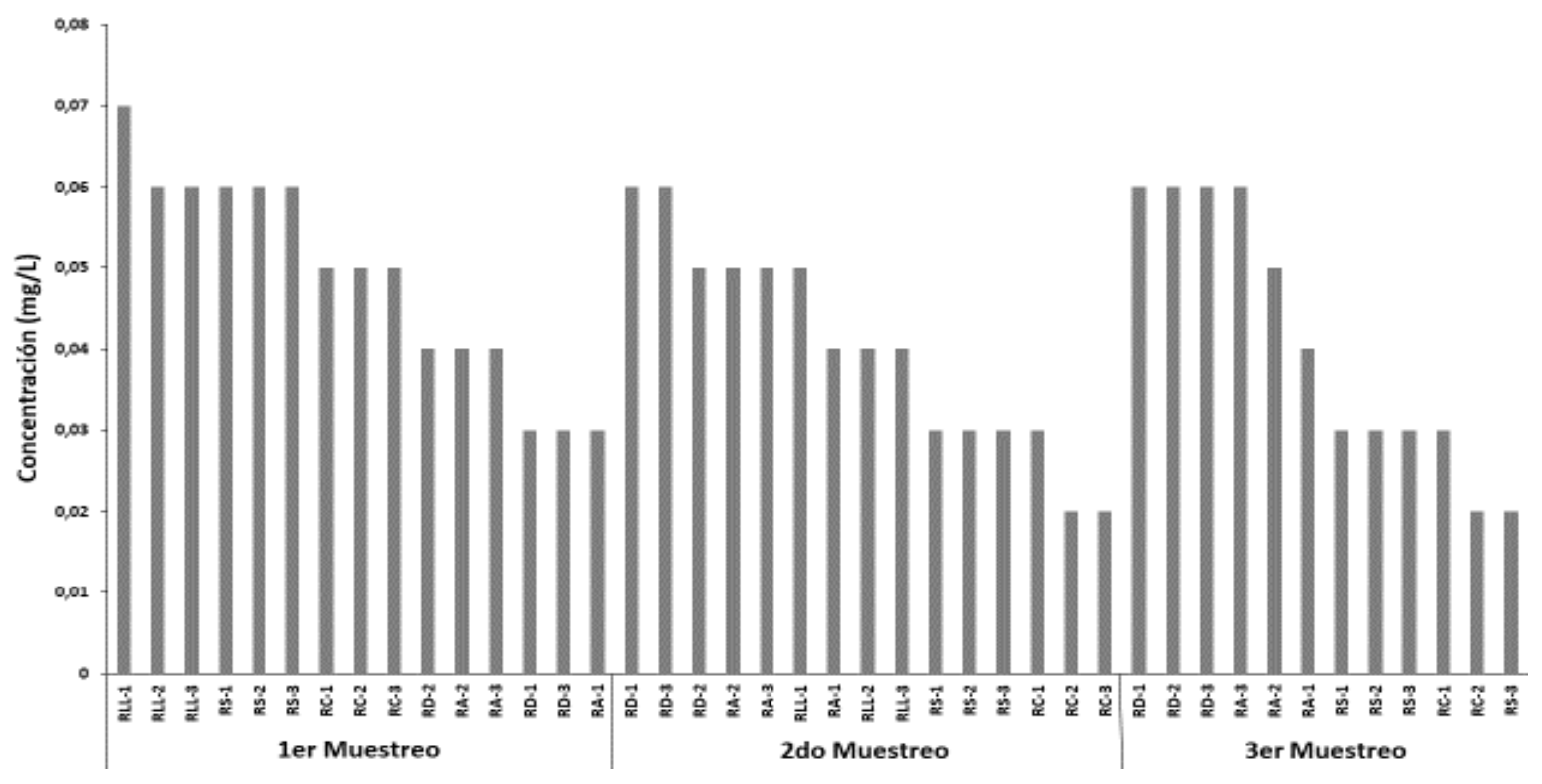


Determinación de cobre (Cu) La Figura 7 denota que en general, todos los puntos de muestreo se encuentran muy por debajo de los 2,o mg/L, límite máximo admisible por la normativa. Se obtuvo un rango de concentraciones de 0,05 mg/L a 0,11 mg/L, en donde el punto de muestreo localizado en la parte alta del río Llorona (RLL-1) mostró la mayor concentración. La concentración promedio de los ríos fue de o,o6 mg/L, este valor se encuentra dentro de lo permitido por la normativa ambiental de calidad de aguas superficiales y zonas costeras, para ríos en preservación, que establece $0,2 \mathrm{mg} / \mathrm{L}$ como límite máximo de concentración de $\mathrm{Cu}$ (Ministerio de ambiente y recursos naturales, 2012). También cumple con el criterio de aceptación, de acuerdo con el reglamento para la evaluación y clasificación de Cuerpos de agua superficiales de Costa Rica (MINAE, 2007), el cual establece un valor de $0,5 \mathrm{mg} / \mathrm{L}$ como valor máximo recomendado para la conservación del equilibrio natural y desarrollo de la vida acuática. La presencia de cobre en el agua natural obtenida, generalmente, se relaciona a la presencia de óxidos o sulfuros que se encuentran en la corteza terrestre y, a consecuencia de fenómenos como la escorrentía, lixiviación, erosión y el contacto del agua, se solubilizan y se pueden encontrar sales de cobre disueltas en aguas superficiales y en mantos acuíferos (Escrig y Morell, 1996).

En aguas superficiales no contaminadas se pueden detectar concentraciones entre los 0,00005 y 0,2 mg/L de cobre, sin embargo, su dinámica ambiental depende de múltiples actores, entre ellos la minería, las características geoquímicas de la región y la industrialización, lo cual permite encontrar concentraciones mayores a $0,05 \mathrm{mg} / \mathrm{L}$ (De Alba, 2007); así, por ejemplo, un estudio realizado por Herrera, Rodríguez, Rojas, Herrera y Chaves, durante los años 2006 y 2010, después de un análisis químico de la calidad de las aguas superficiales de la subcuenta del río Virilla, encontró una concentración de hasta 25,0 mg/L en la microcuenca del río Torres, un valor muy elevado (Valverde, 2013).

Figura 7. Caracterización del metal Cobre en muestras de agua superficial en los ríos Sierpe, Drake, Agujitas, Claro y Llorona, en tres campañas de muestreo realizadas entre 2019-2020.

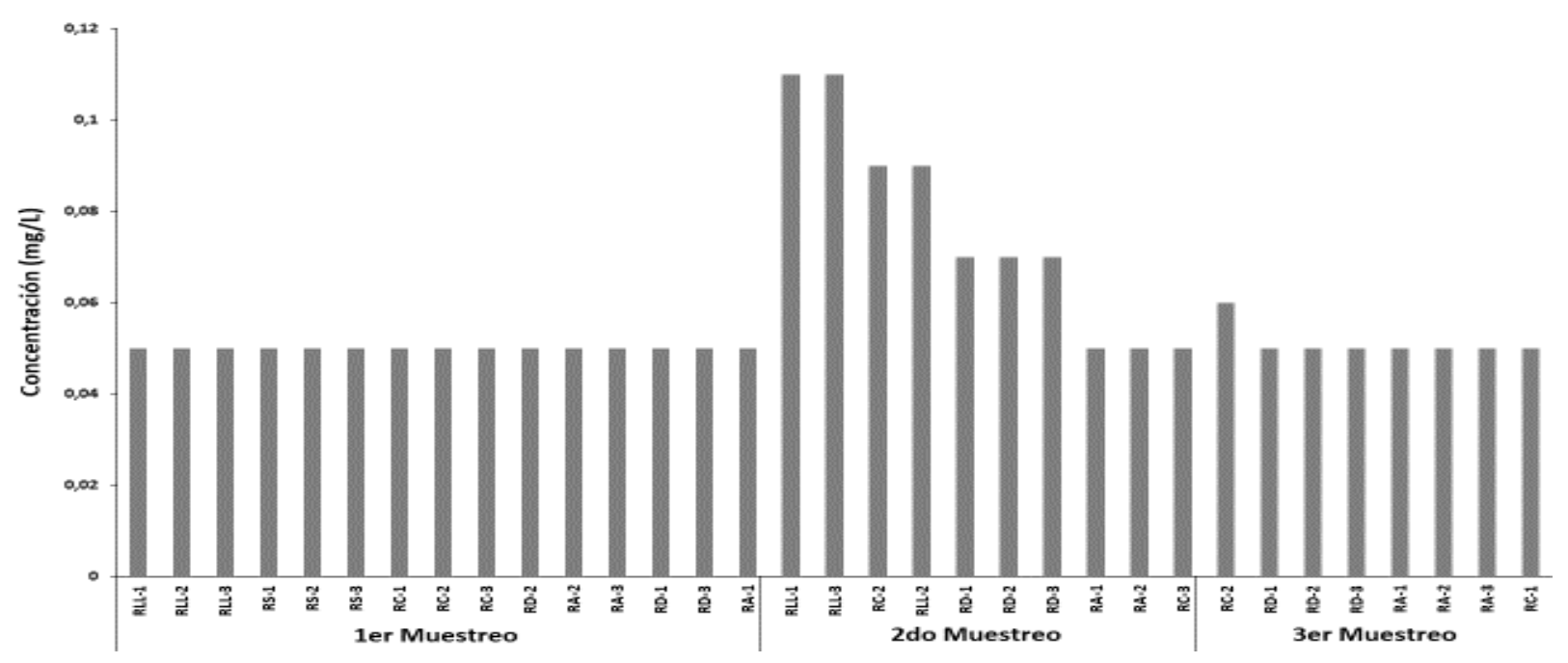


Caracterización de $\mathrm{Pb}, \mathrm{Mn}, \mathrm{As}, \mathrm{Hg}$, Cr y Cd. Las concentraciones de metales pesados en todas las muestras analizadas se encuentran por debajo de los límites de detección del equipo de análisis, datos que se exponen en la siguiente tabla:

Tabla 1. Límites de detección del equipo y límites máximos admisibles para la concentración de metales trazas según Reglamento N³3903 MINAE-S.

\begin{tabular}{|l|l|l|}
\hline Metal Pesado & Límite de detección del equipo $(\mathrm{mg} / \mathrm{L})$ & $\begin{array}{l}\text { Límite máximo admisible, concentración } \\
(\mathrm{mg} / \mathrm{L})\end{array}$ \\
\hline Arsénico & 0,00005 & 0,01 \\
\hline Cadmio & 0,002 & 0,005 \\
\hline Cromo & 0,02 & 0,05 \\
\hline Manganeso & 0,01 & No especifica \\
\hline Mercurio & 0,00006 & 0,001 \\
\hline * Plomo & 0,05 & 0,03 \\
\hline
\end{tabular}

(*) Limite de detección del equipo mayor al límite máximo admisible para la concentración, según el Reglamento 33903 MINAE-S.

\subsection{Hechos relevantes vistos en campo e integración de los resultados}

En general, las concentraciones presentes en los cuerpos de agua analizados no pueden considerarse peligrosas para la conservación y desarrollo de la vida acuática, pues no llegan a superar las concentraciones máximas admisibles establecidas en la Reglamentación para la evaluación y clasificación de calidad de cuerpos de agua superficiales (MINAE, 2007), exceptuando las mediciones de las desembocaduras.

Autores como Mainstone y parr, (2002) denotaron la obligación que tienen las instituciones de conservación de brindar mayor énfasis al control de los niveles iones mayoritarios y metales pesados en las zonas costeras, es importante tener en cuenta que su control no solo es relevante en los ríos, sino también en cuerpos de agua estáticos o humedales y zonas costeras alimentados por ríos.

A pesar de que, en esta zona del Pacífico sur, existe una densidad poblacional relativamente baja, hay un impacto ambiental debido al incumplimiento de buenas prácticas agropecuarias, así como al irrespeto -en la mayoría de las fincas y terrenos- de las áreas de protección de los ríos, que debe tenerse presente, según la Ley Forestal, es de 15 metros, en zonas rurales y de 50 metros si el terreno es quebrado. Durante los muestreos fue notorio ver desechos sólidos -envolturas y botellas de fertilizantes y plaguicidas provenientes de las zonas agrícolas- en la parte alta de los ríos de la zona de estudio; que por escorrentía, los usos indiscriminados de estas sustancias pueden alcanzar el río fácilmente.

La ganadería extensiva desarrollada en la zona media y baja de la cuenca evidenció una afectación tangible de desechos orgánicos sobre los cuerpos de agua, que favorecen procesos de crecimiento de algas y la pérdida de suelos por procesos erosivos, aumento de la demanda de oxígeno disuelto en el agua y, consecuentemente, la generación de procesos eutróficos consolidados, los cuales se logran ubicar en varios tramos de los ríos en zonas bajas, en donde existen, incluso, indicadores edáficos como el azolve en los linderos de los ríos; tal evidencia motiva, además, a realizar otros estudios con los sedimentos. 


\section{Conclusiones}

De los ríos estudiados, el río Llorona fue el que presentó valores más altos de iones mayoritarios y minoritarios disueltos en sus aguas, también el que presentó una concentración de cloruros y sulfatos más alta a lo largo de su cauce; tal situación refleja que es uno de los ríos más vulnerables a sufrir procesos de contaminación ambiental, por lo que se recomienda mantener este tipo de monitoreo periódico sobre el río.

Los ríos Drake, Llorona y Agujitas son los tres ríos que presentan concentración iónica, en general, más alta, provocada principalmente por las actividades agropecuarias en la zona. Tal situación favorece el desarrollo de procesos eutróficos en la zona media y baja, los ríos Claro y Sierpe, que se ubican en zonas menos pobladas y con bosques altamente consolidados, no tienen un impacto ambiental notorio en la calidad del agua.

Los datos analíticos obtenidos propician una gestión hídrica en el Pacífico Sur, la cual busca mantener su conservación y brindar un aprovechamiento sostenido de los recursos, con miras a proteger los ecosistemas y la diversidad de la zona de estudio. El mal manejo durante las actividades antropogénicas propicia impactos ambientales evidentes; por ejemplo, se notó un crecimiento desmedido de algas verdes en el epilimnio debido a la existencia de una alta concentración de macronutrientes, existentes, posiblemente, por el uso excesivo de fertilizantes y por microorganismos vivos que conduce a cambios fisicoquímicos y biológicos de los cuerpos de agua. También la pérdida de suelo por la erosión, provocada por la ganadería extensiva, aumenta la solubilidad por escorrentía de iones y la consecuente pérdida de fertilidad de los suelos.

Los ríos Llorona, Drake, Agujitas, Claro y Sierpe, según los resultados obtenidos, son ríos saludables desde el punto de vista fisicoquímico, pero presentan zonas vulnerables debido a la actividad antrópica. El impacto ambiental en los ríos es tangible, sin embargo, la capacidad de carga del ecosistema acuático en aguas dulces superficiales y, por consiguiente, el de recuperación del ecosistema en esta zona rural, permite una mejora sustancial durante la estación lluviosa. Tal recuperación ocurre gracias al factor de dilución y a la capacidad de oxidación de la carga másica orgánica que transita sobre los ríos, en donde se ve favorecida la oxigenación del agua a lo largo de la cuenca y, por ende, se aceleran los procesos de depuración.

Se puede afirmar, según los resultados, que las aguas superficiales en las zonas altas y medias de los ríos estudiados son blandas; sin embargo, los procesos de intrusión salina, erosión y pérdida de suelo en la zona bajas cercanas a la costa convierten el agua en la parte baja de la cuenca en aguas duras, principalmente en los alrededores de las desembocaduras, haciéndolas no aptas para potabilización y limitando el abastecimiento para uso y consumo humano en dichos lugares.

Finalmente, ninguno de los metales trazas analizados sobrepasan una concentración que sea de riesgo para la salud de las personas o para la salud del ecosistema, por ende, no existe evidencia científica que permita suponer procesos de bioacumulación en la cadena trófica en la zona de estudio.

\section{Referencias Bibliográficas}

Acuña-González, J., Vargas-Zamora, J. A., Gómez-Ramírez, E., y García-Céspedes, J. (2004). Hidrocarburos de petróleo, disueltos y dispersos, en cuatro ambientes costeros de Costa Rica. Revista de Biología Tropical, 52, 43-50. http://www.scielo. sa.cr/scielo.php?script=sci_arttext\&pid=Soo34774420040006oooo5\&lng=en\&nrm=iso\&tlng $=\mathrm{es}$

Alvarado, J. J., Beita-Jiménez, A., Mena, S., FernándezGarcía, C., \& Guzmán-Mora, A. G. (2015). Osa conservation area (Costa Rica) coral ecosystems: Structure and conservation needs [Ecosistemas coralinos del Área de Conservación Osa, Costa Rica: Estructura y necesidades de conservación]. Revista de Biologia Tropical, 63(April), 219-259. https://doi.org/10.15517/rbt.v63i1.23105 
Arellano, F. y Vargas, A. (2000). Casos De Contaminación Por Intrusión Salina En Acuíferos Costeros De La Península De Nicoya (Costa Rica). Revista geológica de América Central. o(25), 8. https:// revistas.ucr.ac.cr/index.php/geologica/article/ view/8540/8065

Ashraf, M. A., Maah, M. J., y Yusoff, I. (2010). Water Quality Characterization of Varsity Lake. CODEN ECJHAO E-Journal of Chemistry, 7, 973-4945. http://www.e-journals.net

AWWA. (1999). Standard Methods for the Examination of Water and Wastewater Part 1000 Standard Methods for the Examination of Water and Wastewater. American Water Works Association, 1-541.

Beita, W. (2008). "Caracterización fisicoquímica de las aguas superficiales de la cuenca del río Rincón en la Península de Osa, Puntarenas, Costa Rica.”

Bolaños-Alfaro, J. D., Cordero-Castro, G., y SeguraAraya, G. (2017). Determinación de nitritos, nitratos, sulfatos y fosfatos en agua potable como indicadores de contaminación ocasionada por el hombre, en dos cantones de Alajuela (Costa Rica). Revista Tecnología En Marcha, 3o(4), 15. https:// doi.org/10.18845/tm.v3oi 4.3408

Casado, J., Calvo, D., Monterde, E., Alfonso, P. (2013). CUENCA DEL LLOBREGAT EN EL BAGES. 287-296.

Costa Rica Poder Ejecutivo. (2015). Reglamento para la Calidad del Agua Potable $N^{\circ} 38924-S$. In Diario Oficial La Gaceta (Vol. 1, Issue 170). http://www.pgrweb.go.cr/scij/Busqueda/ Normativa/Normas/nrm_texto_completo. aspx? param1 $=$ NRTC \&nValor $1=1 \&$ nValor 2 $=80047 \&$ nValor $3=101480 \&$ strTipM $=$ TC
Cravo, A. (2017). Nutrient concentrations in coastal waters: Impact of the Guadiana River. Ciencias Marinas, 29(4), 483-495. https://doi.org/10.7773/ cm.v29i4. 167

Dávila, P. M., y Valdés, J. (2015). Variabilidad temporal de las masas de agua costeras en bahía San Jorge. Antofagasta, Chile $23^{\circ} \mathrm{S}$ ( 2008-2012 ). 5o, 61-80. https://doi.org/10.4067/So71819572015000100006

De Alba, M. I. D. (2007). Determinación de subtrazas de cobre en aguas naturales previa preconcentración con discos de SDB Modificados. https://rodin. uca.es/bitstream/handle/10498/17203/ Trabajo Fin De Máster Margarita Díaz de Alba. pdf?sequence $=1$ \&isAllowed $=\mathrm{y}$

Escrig, I., y Morell, I. (1996). Origen y comportamiento de $\mathrm{Cd}, \mathrm{Cr}, \mathrm{Cu}, \mathrm{Pb}$ y $\mathrm{Zn}$ en el subsistema acuifero de la Plana de Castellon. Estudios Geologicos, 52(5-6), 259-268.

García-Avilés, J. (2002). Biodiversidad de los humedales del Parque Regional del Sureste. II. Heterópteros acuáticos. In Centro de Investigaciones Ambientales de la Comunidad de Madrid "Fernando González Bernáldez” (Issue January).

Grunberger, O y Janeau, J. (1991). Geoquímica de las lluvias, de las aguas de escurrimiento.

La Gaceta. (2007). Diario Oficial La Gaceta N. 178, lunes 17 de setiembre 2007. https://www.aya. go.cr/centroDocumetacion/catalogoGeneral/ Reglamento evaluación y clasificación de calidad de cuerpos de agua superficiales.pdf

Lazo-Páez, A., y Solís-Castro, Y. (2019). Interpretación de Calidad de Agua para Casos con Potencial Intrusión Salina. Revista Tecnología En Marcha, 32, 82-96. https://doi.org/10.18845/ tm.v32i10.4883 
Mainstone, C. P., y Parr, W. (2002). Phosphorus in rivers - ecology and management. Science of The Total Environment, 282-283, 25-47. https://doi. org/10.1016/Soo48-9697(01)0o937-8

Martinez de Victoria, E. (2016). Calcium, essential for health. Nutrición Hospitalaria, 33(4), 26-31. https://doi.org/10.2096o/nh.341

Ministerio de medio ambiente y recursos naturales. (2012). Norma-Ambiental-de-Calidad-de-AguasSuperficiales-y-Zonas-Costeras. Republica dominicana.

Morales-Ramírez, Á. (2011). La diversidad marina del golfo dulce, pacífico sur de costa rica: amenazas a su conservación. Biocenosis, 24, 9-20.

Morales-Ramírez, Á., Acuña-González, J., Lizano, O., Alfaro, E., y Gómez, E. (2015). Rasgos oceanográficos en el Golfo Dulce, Pacífico de Costa Rica: Una revisión para la toma de decisiones en conservación marina. Revista de Biologia Tropical, 63(April), 131-16o. https://doi. org/10.15517/rbt.v63i1.23100

Palter, J., y Ballestero, D. (2007). The distribution of nutrients, dissolved oxygen and chlorophyll. 55(June), 427-436.

Beita-Sandí, Wilson; Barahona-Palomo, Marco. (2011). Fisico-química de las aguas superficiales de la Cuenca del río Rincón, Península de Osa , Costa Rica.

Rosas, H. (2001). Cloruros. Estudio de La Contaminación Por Metales Pesados En La Cuenca Del Llobregat, 2-3. http://www.tesisenred.net/bitstream/ handle/10803/6978/04ComponentesAguaso2. pdf?sequence $=5$

Standard, I. (2006). INTERNATIONAL STANDARD. 2006.
Valverde, R. (2013). El agua en Costa Rica. Ambientales, 45, 124. http://www.ambientico.una.ac.cr/pdfs/ ambientales/45.pdf

Wongsasuluk, P., Chotpantarat, S., Siriwong, W., y Robson, M. (2014). Heavy metal contamination and human health risk assessment in drinking water from shallow groundwater wells in an agricultural area in Ubon Ratchathani province, Thailand. Environmental Geochemistry and Health, 36(1), 169-182. https://doi.org/10.1007/ s10653-013-9537-8 